\title{
Synergistic protection against hyperoxia-induced lung injury by neutrophils blockade and EC-SOD overexpression
}

\author{
Jae H Min ${ }^{1}$, Champa N Codipilly², Sonya Nasim², Edmund J Miller ${ }^{2,3}$ and Mohamed N Ahmed ${ }^{2,4^{*}}$
}

\begin{abstract}
Background: Oxygen may damage the lung directly via generation of reactive oxygen species (ROS) or indirectly via the recruitment of inflammatory cells, especially neutrophils. Overexpression of extracellular superoxide dismutase (EC-SOD) has been shown to protect the lung against hyperoxia in the newborn mouse model. The CXC-chemokine receptor antagonist (Antileukinate) successfully inhibits neutrophil influx into the lung following a variety of pulmonary insults. In this study, we tested the hypothesis that the combined strategy of overexpression of EC-SOD and inhibiting neutrophil influx would reduce the inflammatory response and oxidative stress in the lung after acute hyperoxic exposure more efficiently than either single intervention.

Methods: Neonate transgenic (Tg) (with an extra copy of hEC-SOD) and wild type (WT) were exposed to acute hyperoxia ( $95 \% \mathrm{FiO}_{2}$ for 7 days) and compared to matched room air groups. Inflammatory markers (myeloperoxidase, albumin, number of inflammatory cells), oxidative markers (8-isoprostane, ratio of reduced/ oxidized glutathione), and histopathology were examined in groups exposed to room air or hyperoxia. During the exposure, some mice received a daily intraperitoneal injection of Antileukinate.
\end{abstract}

Results: Antileukinate-treated Tg mice had significantly decreased pulmonary inflammation and oxidative stress compared to Antileukinate-treated WT mice $(p<0.05)$ or Antileukinate-non-treated Tg mice $(p<0.05)$.

Conclusion: Combined strategy of EC-SOD and neutrophil influx blockade may have a therapeutic benefit in protecting the lung against acute hyperoxic injury.

Keywords: Extracellular superoxide dismutase, Antileukinate, CXC-chemokine receptor, Hyperoxia, Lung injury

\section{Background}

Supplemental oxygen is a common, and life saving, strategy used in neonatal intensive care units [1]. However, exposure to high concentrations of oxygen causes increased oxidative stress [2-4], inflammation [5,6] and damage to lung tissues [2-7]. Persistent exposure to hyperoxia eventually results in irreversible pulmonary toxicity and death [8]. Preterm infants are particularly vulnerable to oxygen toxicity as a consequence of an immature antioxidant system $[9,10]$. Oxygen may damage

\footnotetext{
* Correspondence: mahmed2@nshs.edu

${ }^{2}$ Center for Heart and Lung Research, Feinstein Institute for Medical Research, Manhasset, NY 11030, USA

${ }^{4}$ Department of Pediatrics, Cohen Children's Medical Center at New York, New Hyde Park, NY, USA

Full list of author information is available at the end of the article
}

lung cells directly via generation of reactive oxygen species (ROS) [11] or indirectly via the action of inflammatory cells and inflammatory mediators [12,13]. These responses, in turn, overwhelm the cellular antioxidant defenses and lead to the accumulation of toxic levels of ROS. Thus, there is a need to develop better treatment strategies to reduce the oxidative burden on the lung, modulate cytokine networks, and prevent recruitment of inflammatory cells that are responsible for the tissue damage.

While supplementing the immature antioxidant system in preterm infants has been investigated, clinical trials of antioxidant vitamin [14] or antioxidant enzyme supplementation [15] have had only modest success or have been ineffective at preventing bronchopulmonary dysplasia (BPD). However, overexpression of manganese 
superoxide dismutase conferred superior survival in hyperoxia-exposed transgenic ( $\mathrm{Tg}$ ) adult mice [16]. Extracellular superoxide dismutase (EC-SOD) is an antioxidant enzyme that scavenges the potentially harmful superoxide free radical and is highly expressed in the extracellular matrix of lung and vascular tissue in humans and mice. Overexpression or administration of EC-SOD has been shown to protect against a variety of pulmonary insults, including exposure to hyperoxia [17-19], lipopolysaccaride (LPS) [20], and influenzainduced lung injury [21]. EC-SOD is also known to inhibit neutrophil influx in response to hyperoxia [18], LPS [20], bleomycin and bacteria [22].

Cellular injury following oxidative stress provokes an inflammatory response. Pro-inflammatory cytokines such as TNF $\alpha$ and IL- $1 \beta$ are released from the alveolar epithelium, resident macrophages, and other cells following cellular injury [12]. Resident alveolar macrophages orchestrate the initial inflammatory cytokine response and contribute to the initiation of neutrophil influx by secretion of neutrophil chemokines such as IL-8, human growth-related oncogene $\alpha(\mathrm{GRO} \alpha)$, complement $5 \mathrm{a}$ (C5a), platelet activating factor, and platelet derived growth factor. The role of cytokines in coordinating the inflammation, have made them a target for potential therapies for BPD. In addition, administration of neutralizing antibody against neutrophil chemokines coincident with intrapulmonary administration of endotoxin has been found to prevent lung injury in rats, regardless of accompanying high level of proinflammatory cytokines [23]. Therefore, the neutrophil recruitment is noted to be a central effector in the early stages of lung inflammation, further stimulating the accumulation of neutrophil chemokines that provide autocrine signaling, enhancing the oxidative stress by releasing large quantities of extracellular free radicals via NADPH oxidase pathway (respiratory burst), and protease secretion.

Auten et al. demonstrated that neutralizing antibodies against the neutrophil chemokine, cytokine-induced neutrophil chemoattractant-1 (CINC-1) prevented abnormal lung development in newborn rats exposed to 95\% $\mathrm{O}_{2}$ [24]. Furthermore, blocking neutrophil influx also ameliorated nucleic acid damage and DNA oxidation in this model [25]. Liao also described that blocking neutrophil influx by using the CXC-chemokine receptor antagonist (SB265610) protected alveolar development and improved lung function in hyperoxia-exposed newborn rats [26].

Antileukinate, a hexapeptide with acetylated aminoterminus and amidated carboxy-terminus (Ac-RRWWCR$\mathrm{NH}$, ) is a potent inhibitor of CXC receptors 1 and 2 [27]. It specifically inhibits neutrophil chemotaxis and degranulation and has been shown to inhibit lung inflammation in a number of animal models and species [28-34]

While both anti-oxidant and anti-inflammatory strategies have been used separately, each individual approach has only produced a partial protection against hyperoxia. This is the first study to examine, in detail, the protective effects on hyperoxia-induced acute lung injury of combining increased EC-SOD production with inhibition of CXCR 1 and 2 to reduce neutrophil activation.

\section{Material and methods}

\section{Animals and care}

All animal use was approved in advance by the Institutional Animal Care and Use Committee at the Feinstein Institute for Medical Research and was conducted in accordance with guidelines set by the US Animal Welfare Act and National Institutes of Health.

\section{hEC-SOD transgenic mice}

Transgenic B6C3 newborn mice expressing an extracopy of human EC-SOD were used. The human gene was under the control of the SP-C promoter targeting hEC-SOD overexpression to alveolar type II and nonciliated distal bronchial epithelial cells $[18,35]$.

$\mathrm{Tg}$ founders were bred onto the $\mathrm{B} 6 \mathrm{C} 3$ strain to obtain several generations of animals and were identified by PCR analysis of tail DNA using the forward primer EC1 (5'-AGACACCTTCCACTCTGAGG-3') and the reverse primer EC2 (5'-GTTTCGGTACAAATGGAGGC-3') [18].

\section{Antileukinate (CXC-chemokine receptor antagonist)}

Antileukinate (Ac-RRWWCR-NH) was synthesized by Multiple Peptide Systems (San Diego, CA) and was administered daily with a single intraperitoneal dose of $100 \mu \mathrm{g} / \mathrm{g}$ in $0.1 \mathrm{ml}$ sodium chloride $(0.9 \% \mathrm{w} / \mathrm{v})$ for 7 days beginning at birth. Antileukinate-non-treated mice received sodium chloride alone in a similar fashion. The dose of Antileukinate was based on our previous studies that found similar concentrations to be effective in reducing neutrophil trafficking, and were non-toxic $[29,34]$. The first dose was given before exposure as soon as litters were born and a total of 7 doses of Antileukinate were given during exposure.

\section{Exposure to acute normobaric hyperoxia and injection of Antileukinate}

Newborn mice (WT and $\mathrm{Tg}$ ) were randomly assigned into 8 groups (10 pups/group) at birth as follows (Table 1).

Two independent experiments were conducted as paired exposures, with one litter receiving $95 \% \mathrm{O}_{2}$ which was continuously monitored using an $\mathrm{O}_{2}$ analyzer, and the other housed in room air. Food and water were available ad libitum. Nursing dams were switched 
Table 1 Classification of the eight studied groups based on their genotype, exposure and antileukinate treatment

\begin{tabular}{lcccccccc}
\hline Group: & $\mathbf{1}$ & $\mathbf{2}$ & $\mathbf{3}$ & $\mathbf{4}$ & $\mathbf{5}$ & $\mathbf{6}$ & $\mathbf{7}$ & $\mathbf{8}$ \\
\hline $\mathrm{WT}$ & + & - & + & - & + & - & + & - \\
$\mathrm{Tg}$ & - & + & - & + & - & + & - & + \\
$21 \%(\mathrm{RA})$ & + & + & - & - & + & + & - & - \\
$95 \% \mathrm{O}_{2}(\mathrm{H})$ & - & - & + & + & - & - & + & + \\
Antileukinate & - & - & - & - & + & + & + & + \\
\hline
\end{tabular}

between room air and oxygen-exposed litters every 24 hours to prevent maternal $\mathrm{O}_{2}$ toxicity. There were no mortalities during the 7 day- hyperoxic exposure.

At 8 days, pups were euthanized with $150 \mathrm{mg} / \mathrm{kg}$ pentobarbital sodium i.p. Lung tissue and bronchoalveolar lavage (BAL), fluid were obtained. One lung from each mouse was used for biochemical assessments and the contra-lateral lung was used for histopathology.

\section{A. Evaluation of the inflammatory markers:}

\section{BAL cell counts}

The trachea was exposed via a midline incision and the lungs were gently lavaged via a tracheal cannula with three aliquots of $0.3 \mathrm{~mL}$ Phosphate Buffered Saline (PBS $0.5 \mathrm{M}, \mathrm{pH}$ 7.4). The volume of the recovered lavage fluid was recorded, and cell counts were determined using a hemocytometer. Differential counts were performed on cells stained with Wright-Giemsa stain as previously described [36]. Samples with gross hemolysis and with $<70 \%$ BAL recovery were not analyzed.

\section{Myeloperoxidase (MPO)}

MPO activity was measured spectrophotometrically in lung tissue homogenates by reaction with $o$-dianisidine using a microplate assay [36-38].

\section{Albumin}

The albumin concentration, in BAL fluid, was measured using the assayMax Mouse ELISA kit (Assaypro, St. Charles, MO), following the manufacturer's instructions.

\section{B. Measurements of oxidative stress:}

\section{8-isoprostane}

Lung tissue was immediately flash frozen in liquid nitrogen at harvest and stored at $-80{ }^{\circ} \mathrm{C}$ until analysis to prevent auto-oxidation. Lipid peroxidation is a well-defined mechanism of cellular damage. 8-isoprostane, an indicator of oxidative stress in vivo was measured in lung tissue homogenates using Oxiselect 8-iso-prostaglandin
F2 $\alpha$ ELISA Kit (Cell Biolabs, Inc., San Diego, CA), following the manufacturer's instructions.

\section{Ratio of oxidized/reduced glutathione}

Reduced and oxidized glutathione was measured in lung tissue homogenates by reaction with DTNB (5, 5'dithiobis-2-nitrobenzoic acid) using the Glutathione Assay Kit (Calbiochem, Gibbsontown, NJ), following the manufacturer's instructions.

\section{Histological morphometric evaluation:}

Harvested lungs were fixed at inflation with $10 \%$ phosphate-buffered formalin at $20 \mathrm{~cm} \mathrm{H}_{2} \mathrm{O}$ pressure for $30 \mathrm{~min}$. After overnight immersion in fixative, the lungs were embedded in paraffin wax, cut into $5 \mu \mathrm{m}$-thick sections, and stained with hematoxylin and eosin. Lungs were examined qualitatively, and a quantitative analysis was performed using light-microscopic morphometry. Five animals from each group were evaluated to determine alveolar surface density and alveolar volume density as estimates of surface area and alveolar number, as described previously [25]. Data from each group are expressed as means $\pm \mathrm{SE}$.

\section{Statistical analysis}

Groups were compared using analysis of variance, and a post hoc Tukey Kramer test was performed to determine statistical differences. $\mathrm{p}$ values less than 0.05 were accepted as significant, assuming an $\alpha$ error $=0.05$ and $\beta$ error $=0.10$.

\section{Results}

The effect of combination of hEC-SOD overexpression and Antileukinate on neutrophil influx into lung after acute hyperoxic exposure

There was marked elevation in both the total number of white blood cells and neutrophils in the BAL fluid following hyperoxic exposure. In addition, there was increased MPO activity in the lung tissues of both Antileukinate-non-treated WT and Tg mice after acute hyperoxic exposure. In WT mice, Antileukinate significantly reduced neutrophil counts and MPO activity $(p<0.05)$ (Figure 1A\&B). Of particular importance, there were significantly less neutrophils and MPO in the BAL of Antileukinate-treated Tg compared to Antileukinate treated WT group after acute hyperoxic exposure $(p<0.05)$.

\section{The effect of combination of hEC-SOD overexpression and Antileukinate on lung permeability after acute hyperoxic exposure}

Hyperoxic insult increased the concentration of albumin within the alveolar space, in both WT and Tg mice 

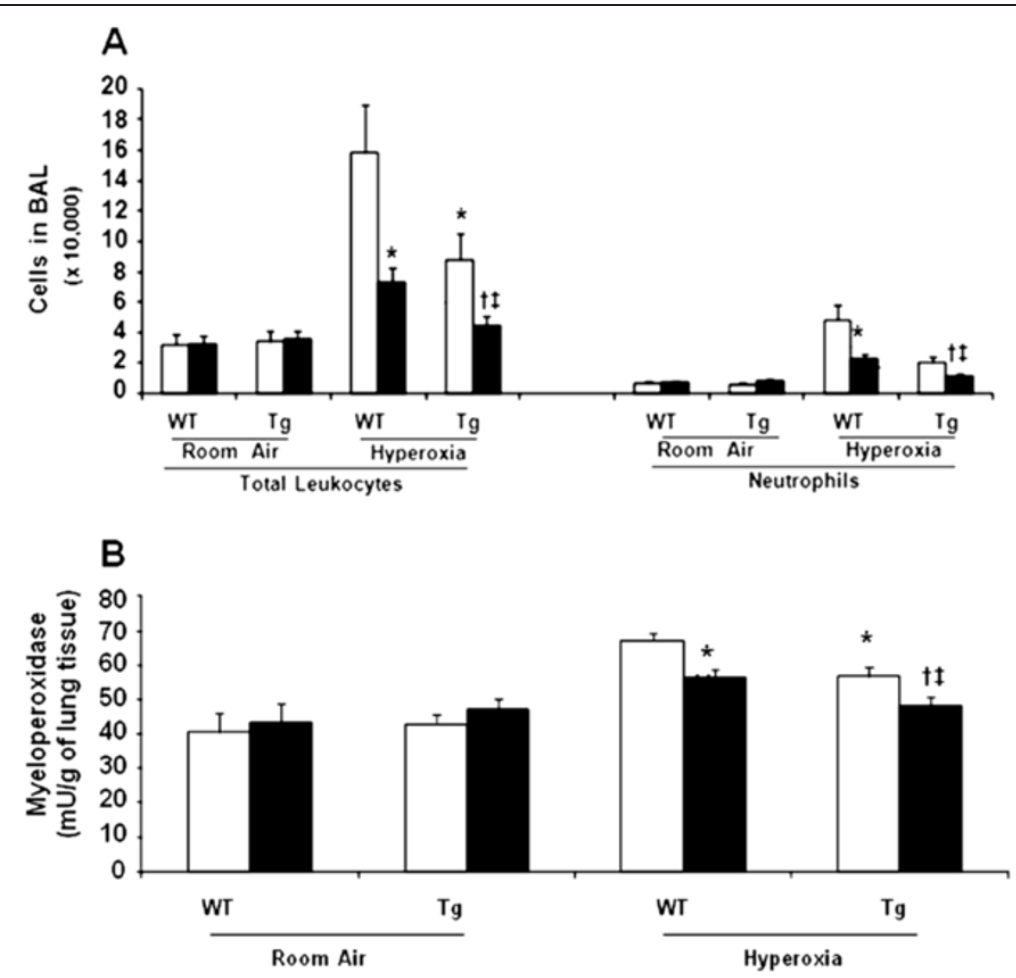

Figure 1 Leukocyte and Myeloperoxidase in BAL (A) Total number of white blood cells and neutrophils in the BAL fluid and (B) MPO concentration in lung tissue of WT and Tg mice \pm Antileukinate ( $\square$ treated, $\square$ non-treated) after 7 day-exposure to air or $95 \% \mathrm{O}_{2}$. Antileukinate significantly decreased total white blood cell and neutrophil counts as well as MPO activity in both WT and Tg mice following $95 \%$ $\mathrm{O}_{2}$ exposure. Tg mice also revealed a significant decrease in total white blood cell and neutrophil count as well as MPO activity compared to WT counterpart groups. Data are mean of ten animals per group \pm SEM. ${ }^{*} p<0.05$ when compared to Antileukinate-non-treated WT/H, ${ }^{\dagger} p<0.05$ when compared to Antileukinate-treated WT/H, ${ }^{\star} p<0.05$ when compared to Antileukinate-non-treated Tg/H.

(Figure 2). In WT mice, Antileukinate significantly reduced the albumin concentration $(p<0.05)$. Antileukinate-treated $\mathrm{Tg}$ mice showed significantly decreased albumin levels compared to the Antileukinate-

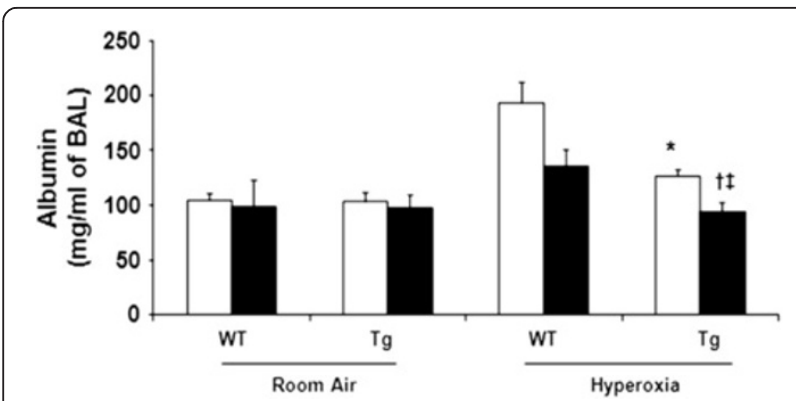

Figure 2 Albumin concentration in BAL fluid of WT and Tg mice \pm Antileukinate ( $\square$ treated, $\square$ non-treated) after 7 day-

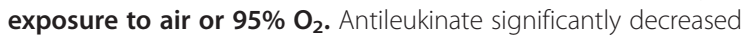
albumin level in both WT and Tg mice following 95\% $\mathrm{O}_{2}$ exposure. Tg mice also revealed a significant decrease in albumin level compared to WT counterpart groups. Data are mean of ten animals per group \pm SEM. * $p<0.05$ when compared to Antileukinate-nontreated WT/H, ${ }^{\dagger} p<0.05$ when compared to Antileukinate-treated WT/H, ${ }^{*} p<0.05$ when compared to Antileukinate-non-treated Tg/H. (RA: room air, H: hyperoxia). treated WT counterpart group after acute hyperoxic exposure $(p<0.05)$.

\section{The effect of hEC-SOD overexpression and Antileukinate} on oxidative stress

8 -isoprostane is an index of oxidative stress in cells. Following exposure to room air, there was no significant difference in 8-isoprostane level in the lung tissue between Antileukinate-treated WT and Tg groups. However, following exposure to $95 \% \mathrm{O}_{2}$, there was marked elevation of 8-isoprostane in both WT and $\mathrm{Tg}$ mice. In WT mice, Antileukinate significantly reduced 8 -isoprostane $(p<0.05)$. In Antileukinate-treated $\mathrm{Tg}$ group, the 8-isoprostane level was further, significantly decreased compared to the Antileukinate-treated Wt counterpart group after acute hyperoxic exposure $(p<0.05)$. In $\mathrm{Tg}$ mice, Antileukinate remarkably decreased the 8-isoprostane level after acute hyperoxic exposure $(p<0.05)$ (Figure $3 \mathrm{~A})$.

The ratio of reduced/oxidized glutathione reflects the oxidative status of tissue. Following exposure to air, there was no significant difference in the ratio of reduced/oxidized glutathione in the lung tissue between Antileukinate-treated WT and Tg mice. Following 


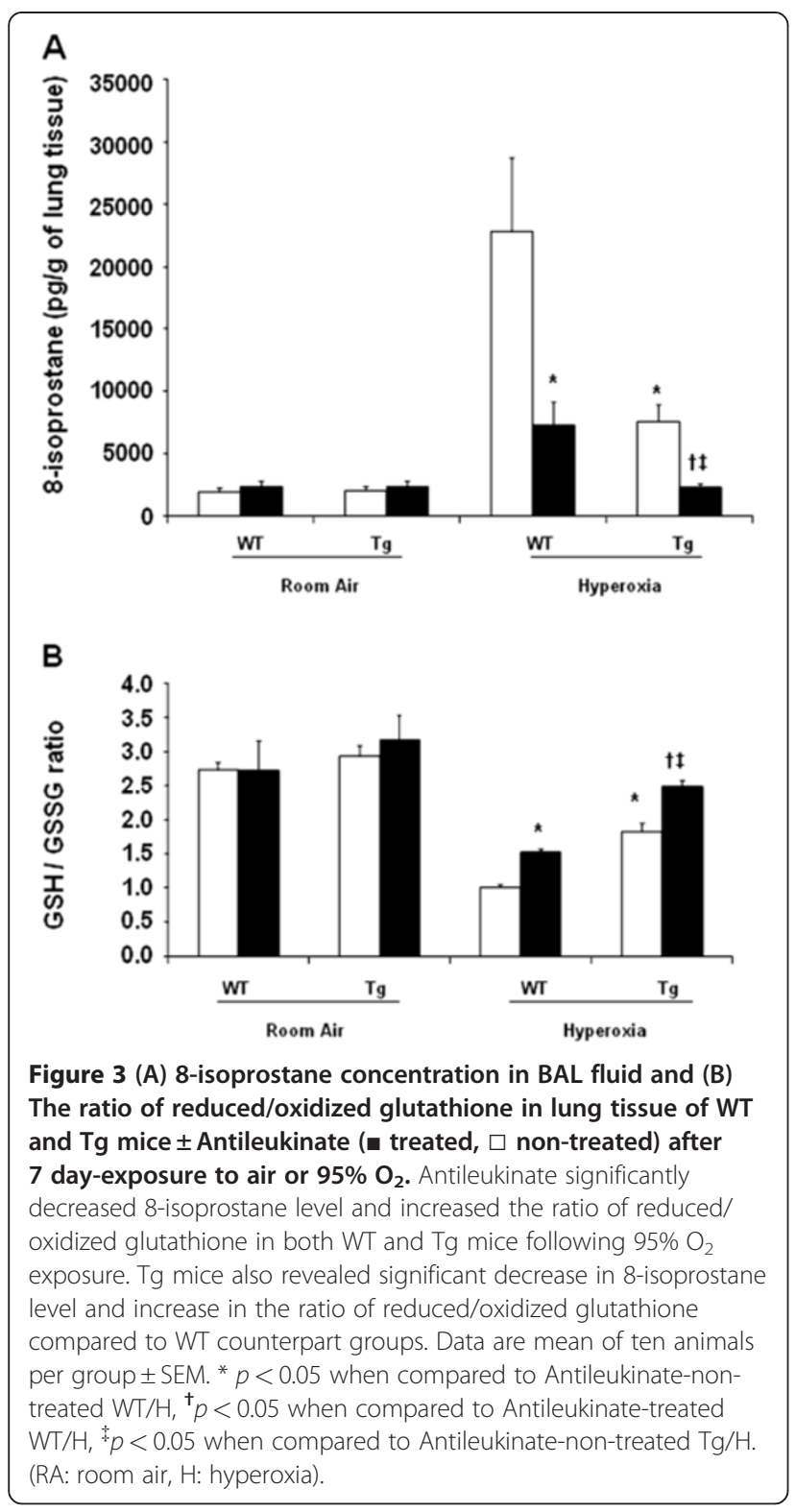

exposure to $95 \% \mathrm{O}_{2}$, there was marked decrease in the ratio in both WT and Tg mice. In WT mice, Antileukinate significantly increased the ratio $(p<0.05)$. The Antileukinate-treated $\mathrm{Tg}$ group showed a further increase in the ratio compared to their Antileukinatetreated WT counterparts after hyperoxic exposure $(p<0.05)$. Notably in Tg mice, Antileukinate revealed significant increase in the ratio after acute hyperoxic exposure $(p<0.05)$ (Figure 3B).

\section{Antileukinate and/or hEC-SOD overexpression improve alveolar development}

Antileukinate significantly improved both alveolar surface and volume density $(p<0.05)$ in WT mice exposed to $95 \% \mathrm{O}_{2}$ for 72 days. However in $\mathrm{Tg}$ mice,
Antileukinate significantly improved alveolar volume density $(p<0.05)$ alone and there was no significant effect on alveolar surface density. Antileukinate-treated $\mathrm{Tg}$ mice also showed significant improvement in alveolar volume density compared to the Antileukinatetreated WT counterpart group following acute hyperoxic exposure $(p<0.05)$. (Figure $4 \mathrm{~A} \& \mathrm{~B})$.

\section{Antileukinate and/or hEC-SOD overexpression reduces alveolar septal thickness inflammatory cellular infiltration after acute hyperoxic lung injury}

Figure 5 represents the histopathology of the lungs from each group. Prominent fibrin deposition as well as inflammatory cell (neutrophil and macrophage) infiltration were observed in the lung sections of hyperoxia Antileukinate-non-treated WT mice (Figures 5C and 6). Other groups demonstrated no evidence of fibrin deposition or inflammatory cell infiltration.

\section{Discussion}

In this study, we tested the hypothesis that the combined strategy of hEC-SOD overexpression and Antileukinate in newborn mice would have a complimentary, protective effect against hyperoxia-induced lung injury. Our data confirmed that when WT and Tg newborn mice were exposed to $95 \% \mathrm{O}_{2}$, Antileukinate significantly decreased inflammation and oxidative stress, reducing acute inflammation and improving alveolar development.

Studies have indicated the link between hyperoxia and inflammation in the developing animal lung. Hyperoxiainduced lung injury can be considered as a bimodal process resulting from direct oxygen toxicity [11] and from the accumulation of inflammatory cells and mediators within the lungs $[12,13]$. ROS can damage the lung cells directly, particularly via lipid peroxidation leading to cellular membrane disruption, followed by cellular necrosis. It is known that hyperoxia results in leakage of ROS from the mitochondrial electron transport chain, from the process of hypoxia/reoxygenation, whereby the conversion of xanthine dehydrogenase to oxidase, and from activated neutrophils recruited to the lungs via NAPDH oxidase pathway. In addition, ROS has been shown to be involved in the recruitment of inflammatory cells by inducing prolonged expression of specific neutrophil binding proteins to endothelial cell surfaces in particular Endothelial Leukocyte Adhesion Molecule-1 (ELAM-1) [39] and Granule Membrane Protein-140 (GMP-140) [40]. Recently, the role of ROS as a signaling molecule in activating NF- $\mathrm{KB}$ has been illuminated [41-43]. In addition to the known role of EC-SOD as a scavenger of free radicals, its anti-inflammatory role has been shown in an animal model of ischemic limb injury [44]. In this model, EC-SOD overexpression leads to 

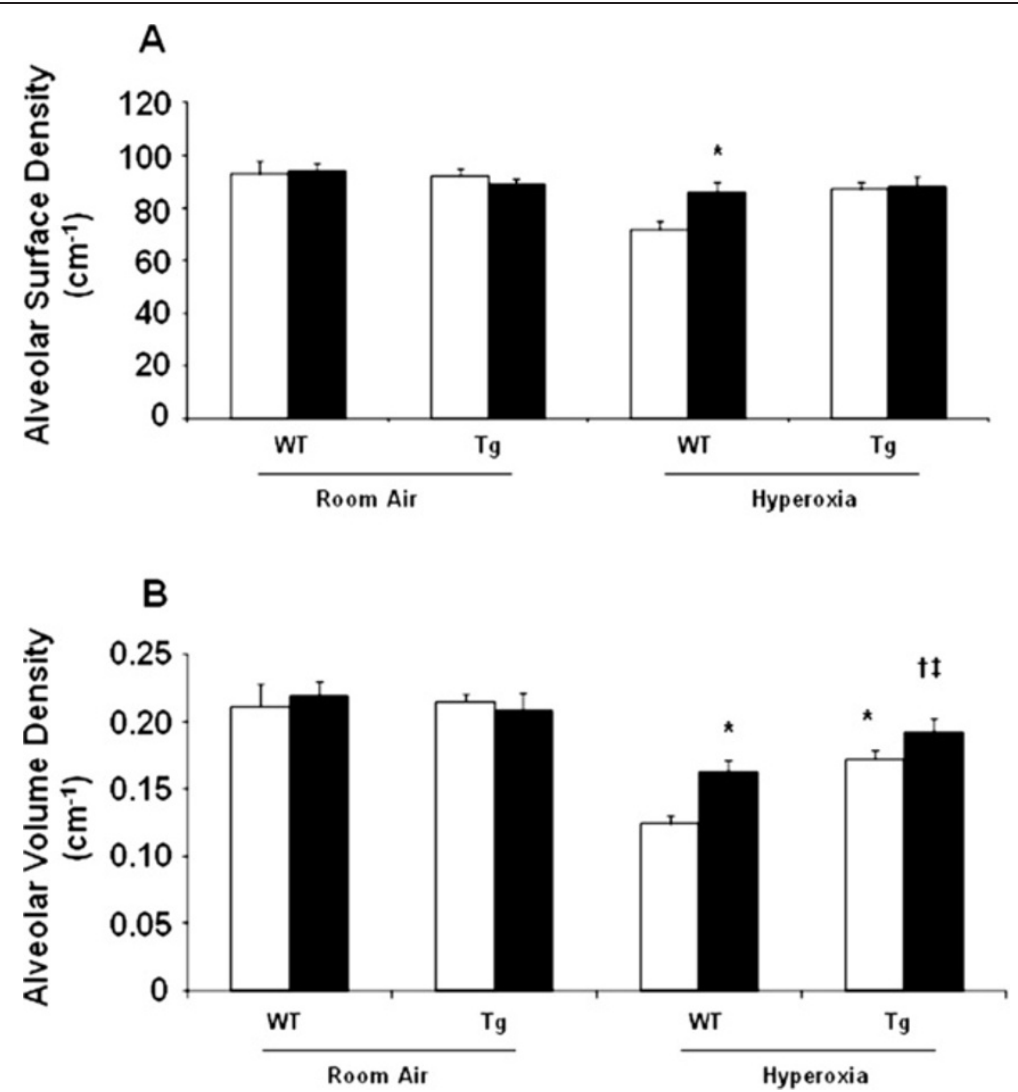

Figure 4 Alveolar surface density (A) and volume density (B) of WT and Tg mice \pm Antileukinate ( $\square$ treated, $\square$ non-treated) after 7 day-

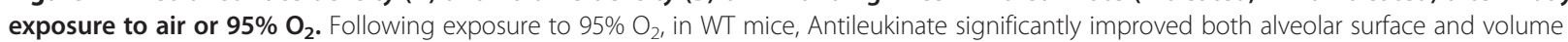
density. In Tg mice, Antileukinate also significantly improved alveolar volume density. However, there was no difference in alveolar surface density. Tg mice also significantly improved alveolar volume density compared to WT counterpart groups after hyperoxic exposure. Data are mean of five animals per group \pm SEM. ${ }^{*} p<0.05$ when compared to Antileukinate-non-treated $\mathrm{WT} / \mathrm{H}^{+}{ }^{\dagger} p<0.05$ when compared to Antileukinatetreated WT/H, ${ }^{\star} p<0.05$ when compared to Antileukinate-non-treated Tg/H. (RA: room air, H: hyperoxia).

reduce expression of TNF $\alpha$, IL1 $\alpha$, IL6, MIP2, and MCP-1 cytokine and VCAM, ICAM, P-selectin, and Eselectin adhesion molecule expressions in injured tissues.

Previous studies have shown that hyperoxia exposed hEC-SOD Tg mice typically exhibit around a two- to three-fold increase in total EC-SOD activity over wildtype littermates $[17,18]$. We have shown previously that augmenting the antioxidant system using hEC-SOD overexpression in newborn mice had a significant impact on hyperoxia-induced lung injury, decreasing the inflammatory response, reducing cell damage and preserving alveolar development [17]. In the present study we have also used direct blockade of the chemokine receptors present on neutrophils. Neutrophils are among the earliest leukocytes to traffic into inflammatory sites and are potent amplifiers of early inflammation, and are recruited to the lung by members of the $\alpha$-chemokine subfamily [45]. Previously, we have shown also that Antileukinate, a hexapeptide with acetylated amino-terminus and amidated carboxyl terminus (Ac-RRWWCR-NH2), blocks both CXCR1 and 2 and inhibits CXC chemokine-induced neutrophil chemotaxis and activation [22]. We and others have shown that Antileukinate reduces neutrophil associated pulmonary inflammation and injury in a variety of models [28,29,31,34].

In WT mice exposed to $95 \% \mathrm{O}_{2}$, Antileukinate significantly improved both alveolar surface and volume density. In $\mathrm{Tg}$ mice exposed to $95 \% \mathrm{O}_{2}$, there was a significant synergistic Antileukinate effect on alveolar volume density but no significant effect on alveolar surface density. This could be attributed to the overestimated alveolar development by edema which developed as result of hyperoxia exposure, which will lead to increase alveolar septal volume and hence alveolar volume density or could be due to short term of hyperoxia exposure (7 days). In the Tg hyperoxic group, the effect of hyperoxia is attenuated by EC-SOD overexpression, which will minimize the edematous inflammatory response and its effect on alveolar volume density. The marked 


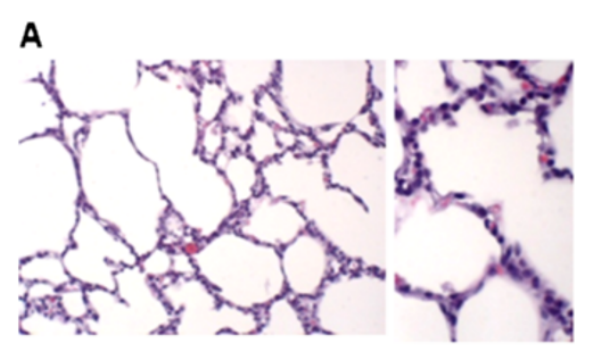

B
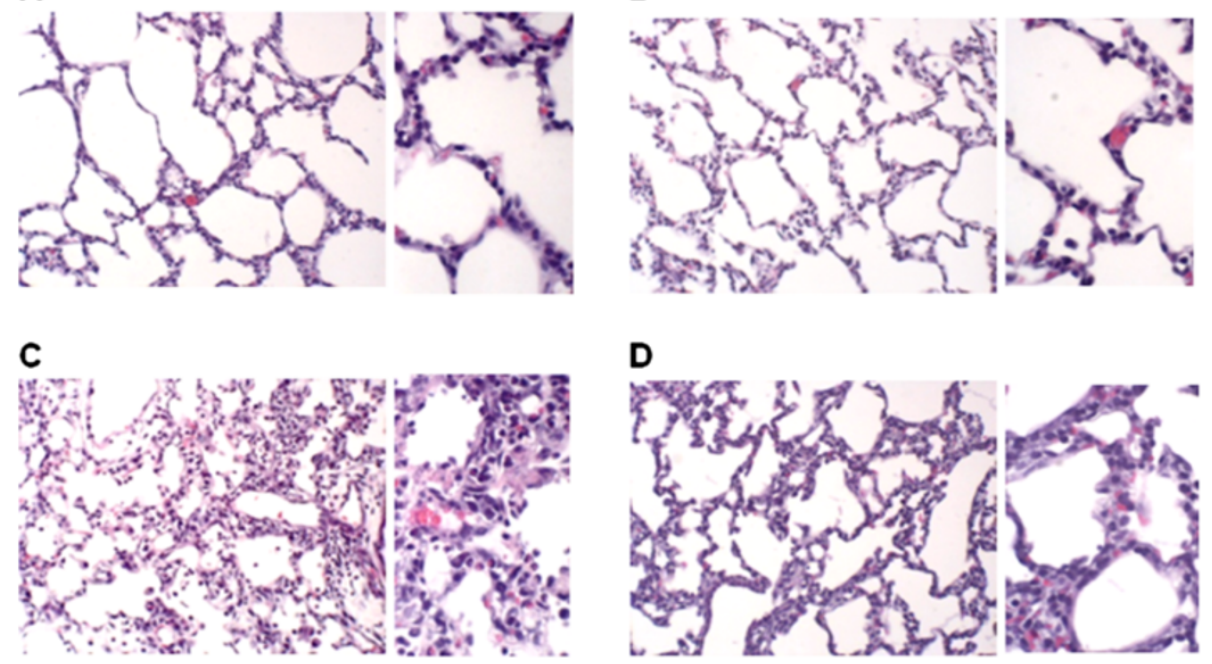

D
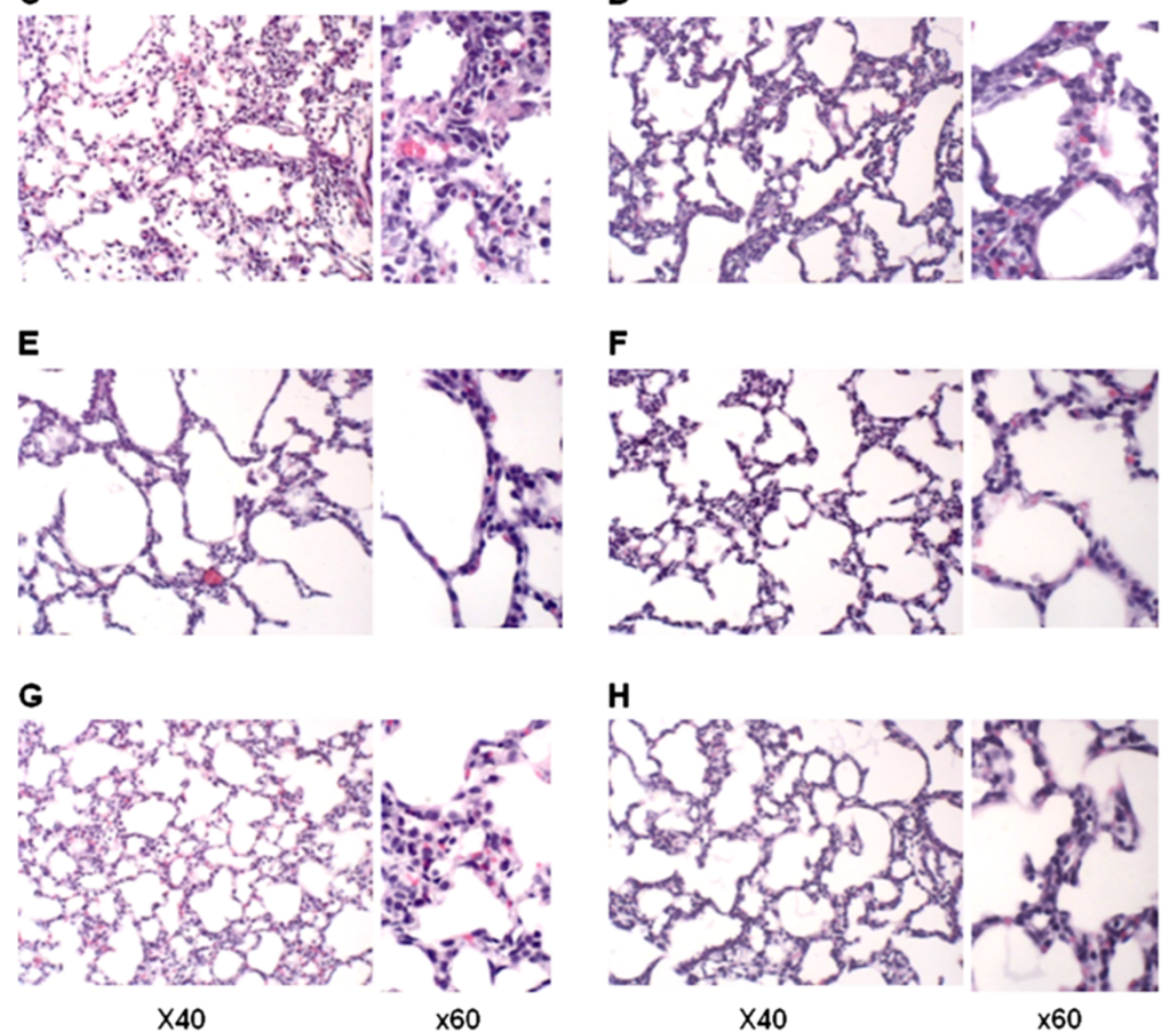

Figure 5 Representative histologic lung sections from WT in room air (A), Tg in room air (B), WT in hyperoxia (C), Tg in hyperoxia (D), Antileukinate-treated WT in room air (E), Antileukinate-treated Tg in room air (F), Antileukinate-treated WT in hyperoxia (G),

Antileukinate-treated Tg in hyperoxia (H). Hematoxylin-Eosin staining. 95\% $\mathrm{O}_{2}$ exposed, Antileukinate-non-treated WT (C) revealed thickened alveolar septum and increased cellularity. Other groups showed no evidence of septal thickness inflammatory cell infiltration.

significant reduction of both alveolar surface and volume density among hyperoxic Wt groups compared to either $\mathrm{Tg}$ alone or treated alone or $\mathrm{Tg}$ and treated with Antileukinate hyperoxia groups, highlight the significant protective effect on lung development, which is completely compromised and arrested in hyperoxic injured lung and eventually leads to development of PBD.

McCord et al. have suggested that when neutrophils are activated in the alveolar spaces, the exposure of the endothelium to superoxide increases dramatically as the adherent neutrophils activate their NADPH oxidase [46]. As the neutrophils degranulate, a variety of enzymes is released including myeloperoxidase and a series of proteases. Activated neutrophils induce partial proteolysis of ECSOD-C, resulting in a diminished affinity for the endothelial surface. Antileukinate inhibits both neutrophil chemotaxis and degranulation [27] thus reducing the peroxidase activity and the proteolytic changes. In addition, Liao et al. have shown that neutrophils are a significant source of hydroxyl radical and that blocking neutrophil influx into the lung could substantially reduce downstream products of reactions with hydroxyl radicals, such as 8-isoprostane [26]. Therefore, a further advantage of reducing neutrophil inflammation, in addition to the reduction of interaction of neutrophil 


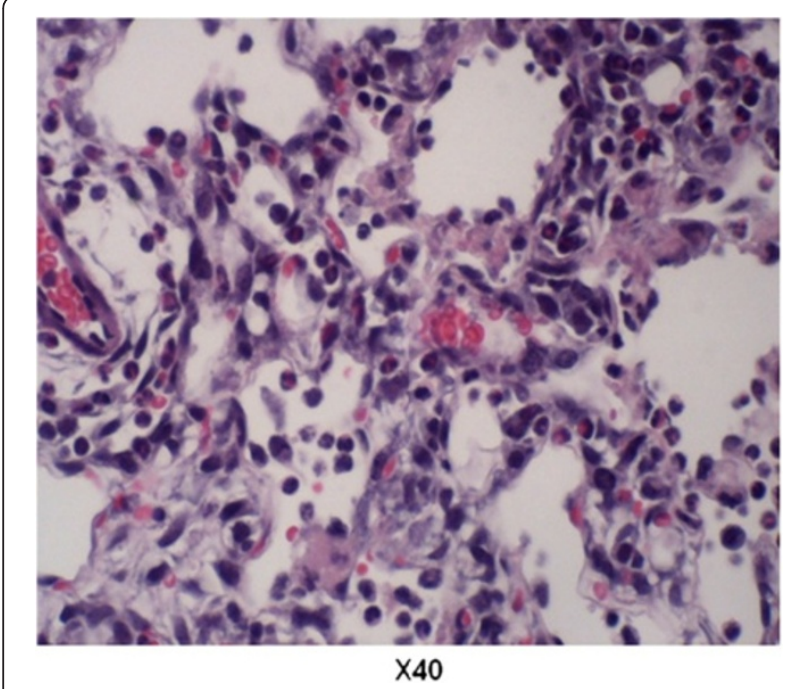

Figure 6 Histologic lung section from $95 \% \mathrm{O}_{2}$ exposed, Antileukinate-non-treated WT mice. Prominent fibrin deposition (arrow) as well as neutrophil (white arrowhead) and macrophage (black arrowhead) infiltration were observed. Hematoxylin-Eosin staining.

components with alveolar cells, is the maintenance of EC-SOD levels and the associated benefits for antioxidative stress. Thus blocking neutrophil influx protects the alveolae, at least in part, by preventing hydroxyl radical accumulation and lipid peroxidation.

While Antileukinate is a hexapeptide with a plasma half life of approximately 45 minutes [47], previous studies have demonstrated effective blockade of neutrophil inflammatory responses using at the daily administration of $20-100 \mu g / g$ [29,31,34]. However, pharmacokinetic studies are needed to determine the optimal dose. In addition, the overall effect of neutrophil blockade on the host immune system is yet to be investigated.

In our current study, we confirmed that overexpression of hEC-SOD or Antileukinate reduced lung inflammation, neutrophil influx and oxidative stress as previous studies have shown. Notably, the level of inflammatory markers and oxidative stress in Antileukinate-treated hEC-SOD transgenic neonatal mice were decreased down to the level of mice on room air. Considering such a complex interplay between ROS, cytokines, and neutrophils, it is not surprising that we can observe dramatic alleviation of lung inflammation when two major pathophysiologic mechanisms of hyperoxic lung injury are targeted.

\section{Conclusion}

The combined strategy of hEC-SOD overexpression and Antileukinate in neonatal mice has an additive protective effect against hyperoxia-induced lung inflammation and reduced oxidative stress in lung tissue after 7 days of hyperoxic exposure. The long term effect of the combined strategy on lung development after hyperoxic exposure still remains to be studied Also the effect of Antileukinate on the immune response and changing the susceptibility of neonates to infection after short and long term treatment approach has to be studied However, we suggest that combination of EC-SOD supplementation and neutrophil blockade can prevent hyperoxia-induced lung injury and may offer an important therapeutic strategy that can protect lung development in premature newborns at risk of developing BPD.

\section{Competing interests}

The authors declare that they have no competing interest.

\section{Author details}

'Department of Pediatrics, State University of New York, Downstate Medical Center, Brooklyn, New York, NY, USA. ${ }^{2}$ Center for Heart and Lung Research, Feinstein Institute for Medical Research, Manhasset, NY 11030, USA. ${ }^{3}$ Department of Medicine, North Shore University Hospital, Manhasset, NY 11030, USA. ${ }^{4}$ Department of Pediatrics, Cohen Children's Medical Center at New York, New Hyde Park, NY, USA. ${ }^{5}$ Department of Pediatrics, Division of Neonatal-Perinatal medicine, Cohen Children, NS-LIJ Hospitals, 300 Community Dr.Manhasset, Manhasset, NY 11725, USA.

\section{Authors' contributions}

$\mathrm{JM}$ : carried out the animal experiment and some molecular and genetics studies and participated in data analysis and drafting the manuscript. CC: carried most of the molecular and genetics studies. SN: participated in genetic testing and microscope study. EM: participated in study design and editing the manuscript. MA: design, statistical analysis, data tabulation and editing the manuscript All Authors read and approved the final manuscript. Grants: NS-LIJ Pediatrics Department

Received: 20 March 2012 Accepted: 20 July 2012

Published: 20 July 2012

\section{References}

1. Bhandari V: Hyperoxia-derived lung damage in preterm infants. Semin Fetal Neonatal Med 2010, 15:223-229.

2. Deuber C, Terhaar M: Hyperoxia in Very Preterm Infants A Systematic Review of the Literature. J of Perinatal \& Neonatal Nursing 2011, 25:268-274.

3. Saugstad OD: Oxygen and oxidative stress in bronchopulmonary dysplasia. J of Perinatal Medicine 2010, 38:571-577.

4. Ciencewicki J, Trivedi S, Kleeberger SR: Oxidants and the pathogenesis of lung diseases. J of Allergy and Clinical Immunology 2008, 122:456-468.

5. Joseph A, Li Y, Koo HC, Davis JM, Pollack S, Kazzaz JA: Superoxide dismutase attenuates hyperoxia-induced interleukin-8 induction via AP-1. Free Radic Biol Med 2008, 45:1143-1149.

6. Rose MJ, Stenger MR, Joshi MS, Welty SE, Bauer JA, Nelin LD: Inhaled Nitric Oxide Decreases Leukocyte Trafficking in the Neonatal Mouse Lung During Exposure to $>95 \%$ Oxygen. Pediatr Res 2010, 67:244-249.

7. Gordo-Vidal F, Calvo-Herranz E, Abella-Alvarez A, Salinas-Gabina I: Hyperoxia induced pulmonary toxicity. Medicina Intensiva 2010, 34:134-138.

8. Folz RJ, Crapo JD: In In Current Pulmonology. Vol 15. Chapter 4. Edited by Tierney DF. Chicago: Mosby Year-Book Inc; 1994:113-136.

9. Saugstad OD: Chronic lung disease: the role of oxidative stress. Biol Neonate 1998, 74(Supl 1):21-28.

10. Rogers S, Witz G, Anwar M, Hiatt M, Hegyi T: Antioxidant capacity and oxygen radical diseases in the preterm newborn. Arch Pediatr Adolesc Med 2000, 154:544-548.

11. Janssen YM, Van Houten B, Borm PJ, Mossman BT: Cell and tissue responses to oxidative damage. Lab Invest. 1993, 69:261-274.

12. Lukacs NW, Ward PA: Inflammatory mediators, cytokines, and adhesion molecules in pulmonary inflammation and injury. Adv Immunol 1996, 62:257-304. 
13. Suttorp N, Simon LM: Lung cell oxidant injury. Enhancement of polymorphonuclear leukocyte-mediated cytotoxicity in lung cells exposed to sustained in vitro hyperoxia. J Clin Invest 1982, 70:342-350.

14. Tyson JE, Wright LL, Oh W, Kennedy KA, Mele L, Ehrenkranz RA, Stoll BJ, Lemons JA, Stevenson DK, Bauer CR, Korones SB, Fanaroff AA: Vitamin A supplementation for extremely-low-birth-weight infants: National Institute of Child Health and Human Development Neonatal Research network. N Engl J Med 1999, 340:1962-1968.

15. Davis JM, Rosenfeld WN, Richter SE, Parad MR, Gewolb $H$, Spitzer AR, Carlo WA, Couser RJ, Price A, Flaster E, Kassem N, Edwards L, Tierney J, Horowitz S: Safety and pharmacokinetics of multiple doses of recombinant human CuZn superoxide dismutase administered intratracheally to premature neonates with respiratory distress syndrome. Pediatrics 1997, 100:24-30.

16. Wispe JR, Warner BB, Clark JC, Dey CR, Neuman J, Glasser SW, Crapo JD, Chang LY, Whitsett JA: Human Mn-superoxide dismutase in pulmonary epithelial cells of transgenic mice confers protection from oxygen injury. J Biol Chem 1992, 267:23937-23941.

17. Ahmed MN, Suliman HB, Folz RJ, Nozik-Grayck E, Golson ML, Mason SN, Auten RL: Extracellular superoxide dismutase protects lung development in hyperoxia-exposed newborn mice. Am J Respir Crit Care Med 2003 167:400-405.

18. Folz RJ, Abushamaa AM, Suliman HB: Extracellular superoxide dismutase in the airways of transgenic mice reduces inflammation and attenuates lung toxicity following hyperoxia. J Clin Invest 1999, 103:1055-1066.

19. Yen CC, Lai YW, Chen HL, Lai CW, Lin CY, Chen W, Kuan YP, Hsu WH, Chen CM: Aerosolized Human Extracellular Superoxide Dismutase Prevents Hyperoxia-Induced Lung Injury. PLoS One 2011, 6(10):e26870.

20. Bowler RP, Nicks M, Tran K, Tanner G, Chang LY, Young SK, Worthen GS Extracellular superoxide dismutase attenuates lipopolysaccharideinduced neutrophilic inflammation. Am J Respir Cell Mol Biol 2004, 31:432-439.

21. Suliman HB, Ryan LK, Bishop L, Folz RJ: Prevention of influenza-induced lung injury in mice overexpressing extracellular superoxide dismutase. Am J Physiol Lung Cell Mol Physiol 2001, 280:L69-L78.

22. Tan RJ, Lee JS, Manni ML, Fattman CL, Tobolewski JM, Zheng M, Kolls JK, Martin TR, Oury TD: Inflammatory cells as a source of airspace extracellular superoxide dismutase after pulmonary injury. Am J Respir Cell Mol Biol 2006, 34:226-232.

23. Tang WW, Yi ES, Remick DG, Wittwer A, Yin S, Qi M, Ulich TR: Intratracheal injection of endotoxin and cytokines. IX. Contribution of CD11a/ICAM-1 to neutrophil emigration. Am J Physiol 1995, 269:653-659.

24. Auten RL, Mason SN, Tanaka DT, Welty-Wolf K, Whorton MH: Antineutrophil chemokine preserves alveolar development in hyperoxia-exposed newborn rats. Am J Physiol Lung Cell Mol Physiol 2001, 281:336-344.

25. Auten $\mathrm{RL}$, Whorton $\mathrm{MH}$, Mason SN: Blocking neutrophil influx reduces DNA damage in hyperoxia-exposed newborn rat lung. Am J Respir Cell Mol Biol 2002, 26:391-397.

26. Liao L, Ning Q, Li Y, Wang W, Wang A, Wei W, Liu X, Auten RL, Tanswell AK, Luo X: CXCR2 Blockade reduces radical formation in hyperoxia- exposed newborn rat lung. Pediatr Res 2006, 60:299-303.

27. Hayashi S, Kurdowska A, Miller EJ, Albright ME, Girten BE, Cohen AB: Synthetic hexa- and heptapeptides that inhibit IL-8 from binding to and activating human blood neutrophils. J Immunol 1995, 154:814-824.

28. Miller EJ, Cohen AB, Peterson BT: Peptide inhibitor of interleukin-8 (IL-8) reduces staphylococcal enterotoxin-A (SEA) induced neutrophil trafficking to the lung. Inflamm Res 1996, 45:393-397.

29. Hayashi S, Yatsunami J, Fukuno Y, Kawashima M, Miller EJ: Antileukinate, a hexapeptide inhibitor of CXC-chemokine receptor, suppresses bleomycin-induced acute lung injury in mice. Lung 2002, 180:339-348.

30. Maus U, von Grote K, Kuziel WA, Mack M, Miller EJ, Cihak J, Stangassinger M Maus R, Schlondorff D, Seeger W: The role of CC chemokine receptor 2 in alveolar monocyte and neutrophil immigration in intact mice. Am Respir Crit Care Med 2002, 166:268-273.

31. Lomas-Neira JL, Chung CS, Grutkoski PS, Miller EJ, Ayala A: CXCR2 inhibition suppresses hemorrhage-induced priming for acute lung injury in mice. J Leukoc Biol 2004, 76:58-64.

32. Lin X, Yang H, Sakuragi T, Hu M, Mantell LL, Hayashi S, Al-Abed Y, Tracey KJ, Ulloa L, Miller EJ: A-Chemokine Receptor Blockade Reduces High Mobility Group Box 1 (HMGB1) Protein Induced Lung Inflammation and Injury and Improves Survival in Sepsis. Am J Physiol Lung Cell Mol Physiol 2005, 289:L583-L590.
33. Bhatia M, Hegde A: Treatment with antileukinate, a CXCR2 chemokine receptor antagonist, protects mice against acute pancreatitis and associated lung injury. Regul Pept 2007, 138:40-48

34. Hirayama S, Shiraishi T, Shirakusa T, Higuchi T, Miller EJ: Prevention of neutrophil migration ameliorates rat lung allograft rejection. Molecular Medicine 2006, 12:208-213.

35. Korfhagen TR, Glasser SW, Wert SE, Bruno MD, Daugherty CC, McNeish JD, Stock JL, Potter SS, Whitsett JA: Cis-acting sequences from a human surfactant protein gene confer pulmonary-specific gene expression in transgenic mice. Proc Natl Acad Sci USA 1990, 87:6122-6126.

36. Zhang YZ, Lin XC, Koga K, Takahashi K, Linge HM, Mello A, Laragione T, Gulko PS, Miller EJ: Strain Differences in Alveolar Neutrophil Infiltration and Macrophage Phenotypes in an Acute Lung Inflammation Model. Molecular Medicine 2011, 17:780-789.

37. Bradley PP, Priebat DA, Christensen RD, Rothstein G: Measurement of cutaneous inflammation: estimation of neutrophil content with an enzyme marker. J Invest Dermatol 1982, 78:206-209.

38. Lundberg C, Arfors KE: Polymorphonuclear leukocyte accumulation in inflammatory dermal sites as measured by $51 \mathrm{Cr}$-labeled cells and myeloperoxidase. Inflammation 1983, 7:247-255.

39. Palluy O, Morliere L, Gris JC, Bonne C, Modat G: Hypoxia/reoxygenation stimulates endothelium to promote neutrophil adhesion. Free Radic Biol Med 1992, 13:21-30.

40. Patel KD, Zimmerman GA, Prescott SM, McEver RP, McIntyre TM: Oxygen radicals induce human endothelial cells to express GMP-140 and bind neutrophils. J Cell Biol 1991, 112:749-759.

41. Rhee SG: Redox signaling: hydrogen peroxide as intracellular messenger. Exp Mol Med 1991, 31:53-59.

42. Thannickal VJ, Fanburg BL: Reactive oxygen species in cell signaling. Am J Physiol Lung Cell Mol Physiol 2000, 279(6):L1005-L1028.

43. Gloire $\mathrm{G}$, Legrand-Poels S, Piette J: NF-kB activation by reactive oxygen species: Fifteen years later. Biochem Pharmacol 2006, 72(11):1493-1505.

44. Laurila JP, Laatikainen LE, Castellone MD, Laukkanen MO: SOD3 reduces inflammatory cell migration by regulating adhesion molecule and cytokine expression. PLoS One 2009, 4(6):e5786.

45. Miller EJ, Cohen AB, Nagao S, Griffith D, Maunder RJ, Martin TR, WeinerKronish JP, Sticherling M, Christophers E, Matthay MA: Elevated levels of NAP-1/interleukin-8 are present in the airspaces of patients with the adult respiratory distress syndrome and are associated with increased mortality. Am Rev Respir Dis 1992, 146:427-432.

46. McCord JM, Gao B, Leff J, Flores SC: Neutrophil-generated free radicals: possible mechanisms of injury in adult respiratory distress syndrome. Environ Health Perspect 1994, 102(suppl 10):57-60.

47. Cohen AB, Miller EJ, Kurdowska AK, Hayashi S, Tuttle R: Methods of inhibiting CXC intercrine molecules. USA:; issued October 12, 1999. Patent No. 5,965,536. http://assignments.uspto.gov/assignments/q?db=pat\&pat=5965536.

doi:10.1186/1465-9921-13-58

Cite this article as: Min et al:: Synergistic protection against hyperoxiainduced lung injury by neutrophils blockade and EC-SOD overexpression. Respiratory Research 2012 13:58.

\section{Submit your next manuscript to BioMed Central and take full advantage of:}

- Convenient online submission

- Thorough peer review

- No space constraints or color figure charges

- Immediate publication on acceptance

- Inclusion in PubMed, CAS, Scopus and Google Scholar

- Research which is freely available for redistribution 